\title{
PAWPAW (Carica papaya) SEEDS POWDER IN NILE TILAPIA (Oreochromis niloticus) DIET \\ 1- GROWTH PERFORMANCE, SURVIVAL, FEED UTILIZATION, CARCASS COMPOSITION OF FRY AND FINGERLINGS
}

Farrag, F. H. ; F. F. Khalil ; A. I. Mehrim and M. M. A. Refaey

Animal Production Dept., Fac. Agric., Al-Mansoura Univ., Al-Mansoura, Egypt

\begin{abstract}
The objective of the present study was to determine the effects of dietary inclusion of different levels of pawpaw seeds powder (PSP) at different periods on the growth performance, survival rate, feed utilization and carcass composition of fry and fingerlings of Nile tilapia (Oreochromis niloticus). The experiment was divided into two periods; the first was feeding period of Nile tilapia fry with PSP for 71 days; and, the second was rearing period where the Nile tilapia fingerlings fed control diet $(0 \mathrm{~g} / \mathrm{kg}$ diet from PSP) for 84 days. Apparently-healthy 1200 Nile tilapia fry of one day old with an average initial body weight $0.012 \mathrm{~g} /$ fry were randomly distributed to 20 treatments. Each treatment was referred to three aquaria. The PSP was added to the basal diet at levels of $0,2,4,6$ and $8 \mathrm{~g} / \mathrm{kg}$ diet for $15,30,45$ and 60 days, different periods.

The results of the first period showed no significant $(P \geq 0.05)$ differences in all growth performance parameters and feed conversion ratio (FCR) during all periods $(15,30,45$ and 60 day); however, different levels of PSP showed significant ( $P \leq$ 0.01 ) improvement in all growth performance, survival (intervals SR and final SR) and feed utilization (FI and FCR) by increasing level of PSP till $6 \mathrm{~g}$ PSP/ $\mathrm{kg}$ diet, then significantly decrease $(P \leq 0.01)$ till $8 \mathrm{~g} P S P / \mathrm{kg}$ diet. In case of the second period, all growth performance parameters and feed utilization (FI and $F C R)$ gradually $(P \leq 0.01)$ improved with increasing feeding periods until 45 day, and then decreased with increasing feeding period (60 day). The interaction between levels of PSP and different periods of treatment indicated that $6 \mathrm{~g} \mathrm{PSP} / \mathrm{kg}$ diet for 45 day had the best $(\mathrm{P}$ $\leq 0.01$ ) values of FW, TWG and ADG compared with other treatments. Thus, the results of this study lead to useful and safety recommended use of $6 \mathrm{~g} \mathrm{PSP} / \mathrm{kg}$ diet for 45 day, to be economic and efficient for fish farms.
\end{abstract}

Keywords: Nile tilapia - Pawpaw seeds powder - fry and fingerlings - growth performance.

\section{INTRODUCTION}

Tilapia species constitute a major and important item in the Egyptian fish farming. It displays many favourable attributes as culture species, on the basis of its general hardness, resistance to diseases, high yield potential and ability to grow on a wide range of natural and cheap artificial foods. Additionally, it also can withstand low oxygen concentrations, overcrowding, tolerate difficult ecological conditions and a wide range of salinities and still produce a highly acceptable flesh (El-Sayed, 2006). Therefore, tilapias are the second only to carps as the most widely farmed freshwater fish in the world (FAO, 2010). In Egypt, the total production of tilapia fish increased from 
Farrag, F. H. et al.

78.35 thousand tons in 1980 to 730.8 thousand tons in 2011, which consider as approximately $53.65 \%$ of the total fish production (GAFRD, 2011).

Generally there are large numbers of feed additives available to improve fish growth performance. Some of these additives, are chemical products (hormones and antibiotics) which may cause unfavorable side effects (Baruah et al., 2008).World Health Organization encourage using of medicinal herbs and plants to substitute or minimize the use of chemicals through the global trend to go back to the nature. Attempts to use the natural materials such as medicinal plants could be widely accepted as feed additives to enhance efficiency of feed utilization and animal productive performance (Levic et al., 2008). The herbs/herbal drugs are used not only against diseases but also as growth promoters, stress resistance boosters and preventatives of infections. Herbs can also act as immunostimulants, conferring the non-specific defense mechanisms of fish and elevating the specific immune response (Pandey et al., 2012). Recently, research has been initiated to evaluate the feasibility of herbal drugs in fish diseases, where the herbal drugs provide a cheaper source for treatment and greater accuracy than chemotherapeutic agents without causing toxicity (Madhuri et al., 2012).

Papaya (C. papaya) is a common human fruit; available throughout the year in the tropic. It is referred to as the "medicine tree" or "melon of health", also papaya is rich with nutrients (Jackwheeler, 2003). It contains medicinal properties and the major active ingredients recorded include, carpine, chymopapain and papain, a bactericidal aglycone of glucotropaeolin, benzyl isothiocyanate, a glycoside sinigrin, the enzyme myrosin, and carpasemine (Jackwheeler, 2003). Seeds of papaya are accounted for about $16 \%$ of the fresh fruit weight (Passera and Spettoli, 1981). According to Bolu et al. (2009), proximate analysis of dried pawpaw seeds contains $97.27 \%$ dry matter, $30.08 \%$ crude protein, $34.80 \%$ crude fat, $1.67 \%$ crude fiber, $7.11 \%$ ash and $23.67 \%$ nitrogen free extract. The seeds contain proteins, carbohydrates, fatty acids, an enzyme carpasemine, and a plant growth inhibitor caricacin (Casey, 1960). The fat content, on a dry weight basis, was $60 \%$ in papaya endosperm (Passera and Spettoli, 1981). Chinoy et al. (1997) reported that oleic, palmitic, stearic and linoleic acids are present in the seeds.

Therefore, Many researchers used pawpaw seeds powder (PSP) as a natural reproductive inhibitor in Nile tilapia (Ekanem and Bassey, 2003; Ekanem and Okoronkwo, 2003; Jegede and Fagbenro, 2008 and Abbas and Abbas, 2011). On the other hand, some of results showed that adding of PSP in diets caused delay of growth and high percentage of mortality in Nile tilapia fry (Ekanem and Okoronkwo, 2003; Ayotunde and Ofem, 2008 and Abbas and Abbas, 2011). Therefore, the present study was conducted to exactly determine the effects of dietary supplementation of different levels of pawpaw seeds powder (PSP) at different periods on the growth performance, survival rate, feed utilization and carcass composition of both Nile tilapia (Oreochromis niloticus) fry and fingerlings. 


\section{MATERIALS AND METHODS}

The present study was carried out in Fish Research Laboratory, Animal Production Department, Faculty of Agriculture, Mansoura University, Dakahlia governorate, Egypt. This experiment was divided into two periods.

The first period (feeding period with PSP):

Apparent-healthy 1200 Nile tilapia (O. niloticus) one day old fry, after absorbing the yolk sac, were obtained from Fish Hatchery, Animal Production Department, Faculty of Agriculture, Mansoura University. Fry was of an average initial body weight of $0.012 \mathrm{~g} /$ fry were randomly distributed to 20 treatments (three aquaria were allocated for one treatment). Fry were stocked at a rate of $30 \mathrm{fry} /$ glass aquarium $(90 \times 40 \times 50 \mathrm{~cm})$. Each aquarium was supplied with $108 \mathrm{~L}$ dechlorinated tap water and an air stone connected to small electric compressor. The experimental design is shown in Table 1.

\section{Experimental diet and feeding:}

Ripe fruits of pawpaw (C. papaya) were obtained from local market in Sharkia government, Egypt, to get the seeds. Then the seeds were cleaned and shade-dried in a drying oven at $50^{\circ} \mathrm{C}$ for 72 hours. The dried seeds were milled into fine particle size $(<250 \mu \mathrm{m})$; and kept in a dry, air-tight transparent plastic container. The chemical analysis of PSP used in the present study was $92.23 \% \mathrm{DM} ; 22.56 \% \mathrm{CP} ; 22.20 \% \mathrm{EE} ; 7.97 \%$ ash and $47.27 \%$ total carbohydrate.

Table 1: Details of the experimental design for treatments in feeding and rearing periods.

\begin{tabular}{|c|c|c|c|}
\hline Treat. & Details & Treat. & Details \\
\hline$T_{1}$ & 0 g PSP / $k g$ diet for 15 day & $T_{11}$ & $4 \mathrm{~g}$ PSP $/ \mathrm{kg}$ diet for 45 day \\
\hline $\mathbf{T}_{2}$ & $0 \mathrm{~g}$ PSP $/ \mathrm{kg}$ diet for 30 day & $T_{12}$ & $4 \mathrm{~g}$ PSP $/ \mathrm{kg}$ diet for 60 day \\
\hline$T_{3}$ & $0 \mathrm{~g}$ PSP $/ \mathrm{kg}$ diet for 45 day & $T_{13}$ & $6 \mathrm{~g}$ PSP $/ \mathrm{kg}$ diet for 15 day \\
\hline $\mathbf{T}_{4}$ & $0 \mathrm{~g}$ PSP $/ \mathrm{kg}$ diet for 60 day & $T_{14}$ & $6 \mathrm{~g}$ PSP $/ \mathrm{kg}$ diet for 30 day \\
\hline$T_{5}$ & $2 \mathrm{~g}$ PSP $/ \mathrm{kg}$ diet for 15 day & $T_{15}$ & $6 \mathrm{~g}$ PSP $/ \mathrm{kg}$ diet for 45 day \\
\hline$T_{6}$ & $2 \mathrm{~g}$ PSP $/ \mathrm{kg}$ diet for 30 day & $T_{16}$ & $6 \mathrm{~g}$ PSP $/ \mathrm{kg}$ diet for 60 day \\
\hline$T_{7}$ & $2 \mathrm{~g}$ PSP $/ \mathrm{kg}$ diet for 45 day & $T_{17}$ & $8 \mathrm{~g} \mathrm{PSP} / \mathrm{kg}$ diet for 15 day \\
\hline$T_{8}$ & $2 \mathrm{~g}$ PSP $/ \mathrm{kg}$ diet for 60 day & $T_{18}$ & $8 \mathrm{~g}$ PSP $/ \mathrm{kg}$ diet for 30 day \\
\hline$T_{9}$ & $4 \mathrm{~g}$ PSP $/ \mathrm{kg}$ diet for 15 day & $T_{19}$ & $8 \mathrm{~g}$ PSP $/ \mathrm{kg}$ diet for 45 day \\
\hline$T_{10}$ & $4 \mathrm{~g}$ PSP $/ \mathrm{kg}$ diet for 30 day & $T_{20}$ & $8 \mathrm{~g}$ PSP $/ \mathrm{kg}$ diet for 60 day \\
\hline
\end{tabular}

The basal diet formulation and chemical analysis are shown in Table 2. The basel diet was prepared by mixing the dry the commercial diet ingredients with oil, before starting the experiment. The PSP was added to the basal diet at levels $0,2,4,6$ and $8 \mathrm{~g} / \mathrm{kg}$ diet. The mash diet was given manually to fry four times daily at 9.00 a.m., 11.00 a.m, 13.00 p.m and 15.00 p.m., for six days a week for examined different periods 15, 30, 45 and 60 day. Fry were fed during the first 45 days at the rate of $30 \%$ of total body weight, and then the rate of feeding was reduced gradually to $15 \%$ until the 71 day (the end of the first period). Because of the difficulty of weighing the fry at this age (stage), the amount of feed in the first week was doubled in the 
second week and three fold in the third week. At the end of the third week, random sample of fry/each aquarium was taken to adjust the feed intake. After that, the fry were weighed biweekly for the adjustment of feed intake, until the end of the first period.

Accumulated wastes were removed from each aquarium two days a week by siphoning of $20 \%$ of the water volume per aquarium, then, equal volume of water was replaced by dechlorinated tap water. The water was aerated by using air pump to permit suitable level of dissolved oxygen for Nile tilapia fry rearing. The dissolved oxygen was in normal range $6-8 \mathrm{mg} / \mathrm{L}$, and water temperature was maintained at $25-27 \stackrel{\circ}{\circ}$ during the experimental period. Light period was controlled to provide a $14 \mathrm{~h}$ light: $10 \mathrm{~h}$ dark daily.

Table 2: Formulation (\%) and chemical analysis of experimental diet at the first and the second periods.

\begin{tabular}{|c|c|c|}
\hline Ingredients & $\begin{array}{c}\text { Experimental diet } \\
\text { composition at } 1^{\text {st }} \text { treated } \\
\text { period supplemented with } \\
\text { PSP }\end{array}$ & $\begin{array}{l}\text { Experimental diet } \\
\text { composition at the } \\
\text { second rearing period }\end{array}$ \\
\hline Fish meal & 46 & 15 \\
\hline Soybean meal & 30 & 35 \\
\hline Wheat bran & - & 16 \\
\hline Crushed corn & 20 & 25 \\
\hline Corn oil & 3 & 5 \\
\hline Molasses & - & 3 \\
\hline Premix $^{1}$ & 1 & 1 \\
\hline \multicolumn{3}{|c|}{ Nutrients composition (\% dry matter basis) } \\
\hline Dry matter (DM) & 92.87 & 89.81 \\
\hline Crude protein (CP) & 37.53 & 27.89 \\
\hline Ether extract (EE) & 5.23 & 6.59 \\
\hline Ash & 10.75 & 11.12 \\
\hline Carbohydrate & 46.49 & 54.40 \\
\hline Gross energy (Kcal / $100 \mathrm{~g} \mathrm{DM})^{*}$ & 451.4 & 442.9 \\
\hline $\begin{array}{l}\text { Protein / energy ratio (mg CP / } \\
\text { Kcal GE) }\end{array}$ & 83.14 & 62.97 \\
\hline
\end{tabular}

1: Premix containing A vit. (15 million l.U.), E vit. (15 mg), B1 vit. (1.0 mg), B12 vit. (5.0 mg),

K3 vit. (2.5 mg), B6 vit. (2.0 mg), Pantothenic acid (10.0 mg), Folic acid (1.2 mg), Biotin (0.05 mg) and D3 vit. (3.0 million I.U.). Copper $(7.0 \mathrm{mg})$, Manganese (100.0 $\mathrm{mg})$, iodine (0.4 mg), Iron (40.0 mg), Zinc (50.0 mg), Selenium (0.15 mg) and anti- oxidant (125.0 mg). ${ }^{*} \mathrm{GE}(\mathrm{Kcal} / 100 \mathrm{~g} \mathrm{DM})=\mathrm{CP} \times 5.64$ + EE x 9.44 + Carbohydrates $\times 4.11$ calculated according to (NRC. 1993).

** $\mathrm{P} / \mathrm{E}$ ratio (mg protein/Kcal gross energy) $=\mathrm{CP} / \mathrm{GE} \times 1000$

\section{The second period (rearing period):}

At the end of the first period, the Nile tilapia fry with an average body weight of $4.674 \pm 1.99 \mathrm{~g}$ were selected from each treatment and stocked at rate of 25 fish / tank (two replicates per treatment) for 84 days rearing period. Each tank ( $1 \mathrm{~m}^{3}$ in volume) was supplied with an upper irrigation open (inlet), an under drainage (outlet), and an air stone connected to electric compressor.

The basal diet formulation and chemical analysis was shown in Table (2). The basel diet was prepared by mixing the dry the commercial diet ingredients with oil, then was pelleted by manufacturing machine (pellets size 
$1 \mathrm{~mm}$ ). During the rearing period diet was given six days a week at a rate of 8 $\%$ of live body weight during the first 4 weeks, and then at a rate of $6 \%$ during the second 4 weeks and at a rate of $5 \%$ until the end of the experimental period. Experimental diets were introduced manually twice daily at 8 a.m. and 14.00 p.m. The amount of feed was adjusted bi-weekly based on the actual fish body weight changes. The water temperature ranged $24-$ $25 \stackrel{\circ}{\circ}$ and photoperiod was controlled by timer provide $14 \mathrm{~h}$ light: $10 \mathrm{~h}$ dark daily during the rearing period.

Individual fish body weight was recorded biweekly to point feed quantity and to calculate growth performance parameters according to Abdelhamid (2000) in form of: average weight gain ( $\mathrm{g} / \mathrm{fish}) \mathrm{AWG}=$ average final weight (g) - average initial weight $(\mathrm{g})$, average daily gain, ( $\mathrm{g} / \mathrm{fish} / \mathrm{day}) \mathrm{ADG}=\mathrm{AWG}$ (g)/experimental period (days), specific growth rate (\%/day) SGR $=[$ In final weight - In initial weight] $\times 100 /$ Experimental period (d), feed conversion ratio $(\mathrm{FCR})=$ feed intake $(\mathrm{g}) /$ live weight gain $(\mathrm{g})$ and survival rate $(\%) \mathrm{SR}=$ end number of the alive fish/the beginning number of the fish $\times 100$. At the end of the experiment, the chemical analyses of the basal diet as well as whole fish body were carried out according to the AOAC (2000).

The data were statistically analyzed by using SAS (2006), with factorial design (5X4) and evaluated by using the following model:

$Y_{i j k}=\mu+L_{i}+B_{j}+L B_{i j}+e_{i j k}$

Where, $Y_{\mathrm{ijk}}$ is the data of growth performance, survival, feed utilization and carcass composition, $\mu$ is the overall mean, $L_{i}$ is the fixed effect of the dietary PSP, $B_{j}$ is the fixed effect of different periods, $L_{i j}$ is the interaction effect between dietary PSP levels with different periods and with $e_{i j k}$ is the random error. The differences between mean were statistically compared for the significance $(P \leq 0.05)$ using Duncan $(1955)$ multiple range test.

\section{RESULTS}

\section{The first period (feeding period with PSP):}

\subsection{Growth performance and feed utilization:}

Growth performance, survival (intervals SR and final SR) and feed utilization (FI and FCR) parameters of Nile tilapia fry fed different levels of PSP for different periods are illustrated in Table 3. The results indicated no significant differences in growth performance parameters and FCR for all periods $(15,30,45$ and 60 day). However, there are significant $(P \leq 0.01)$ differences in SR between all experimental periods, whereas $P_{15}$ (15 day of treatment) recorded the highest SR value compared with other periods. The final SR (60 day) ranged from 77.66 to $84.00 \%$, where the highest $(84 \%)$ and the lowest (77.66\%) final SR were recorded at 15 and 45 day of treatments, respectively. While, FI significantly $(P \leq 0.05)$ increased by increasing the periods.

The results of different levels of PSP showed that all growth performance, survival (intervals SR and final SR) and feed utilization ( $\mathrm{FI}$ and FCR) parameters were significantly increased $(P \leq 0.01)$ by increasing the 
Farrag, F. H. et al.

level of PSP until $\mathrm{L}_{3}(6 \mathrm{~g} \mathrm{PSP} / \mathrm{kg}$ diet), then significantly decreased $(\mathrm{P} \leq$ 0.01 ) by higher level of PSP ( $8 \mathrm{~g}$ PSP / $\mathrm{kg}$ diet). The data revealed also that $\mathrm{L}_{3}$ was the best followed by $\mathrm{L}_{2}(4 \mathrm{~g} \mathrm{PSP} / \mathrm{kg}$ diet). Furthermore, the intervals SR and final SR ranged from 78.75 to $88.75 \%$ and 75.00 to $87.50 \%$, respectively, where the highest values of intervals $S R$ and final $S R$ were recorded in $L_{3}\left(6 \mathrm{~g} \mathrm{PSP} / \mathrm{kg}\right.$ diet) and $\mathrm{L}_{4}(8 \mathrm{~g} \mathrm{PSP} / \mathrm{kg}$ diet), respectively.

Table 3: Overall means of growth performance, survival and feed utilization of Nile tilapia fry fed different levels of PSP for different periods.

\begin{tabular}{|c|c|c|c|c|c|c|c|c|c|}
\hline \multirow{2}{*}{ Treat. } & \multicolumn{5}{|c|}{ Growth performance } & \multicolumn{2}{|c|}{ Survival (\%) } & \multicolumn{2}{|c|}{$\begin{array}{c}\text { Feed } \\
\text { utilization }\end{array}$} \\
\hline & $\begin{array}{l}\text { FW } \\
(\mathrm{mg})\end{array}$ & $\begin{array}{l}\text { TWG } \\
\text { (mg) }\end{array}$ & $\begin{array}{l}\text { ADG } \\
\text { (mg) }\end{array}$ & $\begin{array}{c}\text { RGR } \\
(\%)\end{array}$ & $\begin{array}{l}\text { SGR } \\
(\% / d) \\
\end{array}$ & \begin{tabular}{|c} 
Intervals \\
SR
\end{tabular} & Final SR & $\mathrm{FI}(\mathrm{g})$ & FCR \\
\hline \multicolumn{10}{|c|}{ Period (day) } \\
\hline $\mathbf{P}_{15}$ & 2.68 & 2.66 & 0.038 & 223.6 & 7.51 & $93.30^{A}$ & $84.00^{\mathrm{a}}$ & $2.40^{\mathrm{b}}$ & 1.00 \\
\hline$P_{30}$ & 2.75 & 2.73 & 0.039 & 229.5 & 7.58 & $84.33^{\mathrm{B}}$ & $80.66^{\mathrm{ab}}$ & $2.59^{\mathrm{ab}}$ & 0.97 \\
\hline $\mathbf{P}_{45}$ & 2.79 & 2.77 & 0.039 & 32.8 & 7.60 & $80.66^{\mathrm{B}}$ & $77.66^{\mathrm{b}}$ & $2.58^{\mathrm{ab}}$ & 0.97 \\
\hline$P_{60}$ & 3.03 & 3.01 & 0.042 & 252.9 & 7.69 & $80.66^{\mathrm{B}}$ & $80.66^{\mathrm{ab}}$ & $2.83^{\mathrm{a}}$ & 0.97 \\
\hline$\pm \mathrm{SE}$ & 0.105 & 0.105 & 0.001 & 8.872 & 0.053 & 1.453 & 1.404 & 0.106 & 0.041 \\
\hline P-value & 0.121 & 0.121 & 0.140 & 0.121 & 0.146 & 0.0001 & 0.027 & 0.059 & 0.966 \\
\hline \multicolumn{10}{|c|}{ Level (g PSP/kg diet) } \\
\hline$L_{0}$ & $1.43^{\mathrm{D}}$ & $1.42^{\mathrm{D}}$ & $0.020^{\mathrm{D}}$ & $118.9^{D}$ & $6.72^{\mathrm{D}}$ & $84.16^{\mathrm{A}}$ & $76.66^{\mathrm{B}}$ & $1.56^{\mathrm{C}}$ & $1.14^{\mathrm{A}}$ \\
\hline $\overrightarrow{\mathrm{L}_{1}}$ & $2.53^{\mathrm{C}}$ & $2.52^{\mathrm{C}}$ & $0.036^{\mathrm{C}}$ & $211.7^{\mathrm{C}}$ & $7.52^{\mathrm{C}}$ & $84.96^{\mathrm{A}}$ & $77.91^{\mathrm{B}}$ & $2.51^{B}$ & $1.04^{\mathrm{AE}}$ \\
\hline $\mathrm{L}_{2}$ & $3.42^{\mathrm{A}}$ & $3.41^{\mathrm{A}}$ & $0.048^{A}$ & $286.3^{A}$ & $7.94^{\mathrm{AB}}$ & $78.75^{\mathrm{B}}$ & $75.00^{\mathrm{B}}$ & $2.89^{A}$ & $0.86^{\mathrm{C}}$ \\
\hline $\mathrm{L}_{3}$ & $3.57^{\mathrm{A}}$ & $3.56^{\mathrm{A}}$ & $0.050^{A}$ & $298.7^{A}$ & $8.00^{A}$ & $88.75^{A}$ & $87.50^{A}$ & $2.96^{\mathrm{A}}$ & $0.85^{\mathrm{C}}$ \\
\hline $\mathrm{L}_{4}$ & $3.09^{\mathrm{B}}$ & $3.07^{\mathrm{B}}$ & $0.043^{\mathrm{B}}$ & $257.9^{B}$ & $7.81^{\mathrm{B}}$ & $87.08^{A}$ & $86.66^{A}$ & $3.06^{\mathrm{A}}$ & $1.00^{\mathrm{B}}$ \\
\hline$\pm \mathrm{SE}$ & 0.118 & 0.118 & 0.001 & 9.920 & 0.059 & 1.624 & 1.570 & 0.119 & 0.046 \\
\hline P-value & 0.0001 & 0.0001 & 0.0001 & 0.0001 & 0.0001 & 0.001 & 0.0001 & 0.0001 & 0.0002 \\
\hline
\end{tabular}

Means in the same column having different capital and small letters are significantly different at $(P \leq 0.01)$ and $(P \leq 0.05)$, respectively

The interaction effect between different levels of PSP and different periods on growth performance and feed utilization (FI and FCR) parameters of Nile tilapia fry is presented in Table 4. The results indicated no significant $(P \geq 0.05)$ differences in the intervals $S R$ and final $S R$ among all treatments. The data also indicated that, the Nile tilapia fry treated with $4 \mathrm{~g} \mathrm{PSP} / \mathrm{kg}$ diet for 60 days gave the highest $(P \leq 0.01)$ values of growth performance parameters compared with other treatments. In contrary, the control group $L_{0}$ (0 g PSP $/ \mathrm{kg}$ diet) during all periods had the lowest $(P \leq 0.01)$ growth performance parameters. 
Table 4: The interaction effect between different levels of PSP ( $\mathrm{g} / \mathrm{kg}$ diet) and different periods (day) on growth performance and feed utilization of Nile tilapia fry.

\begin{tabular}{|c|c|c|c|c|c|c|c|c|}
\hline \multirow{2}{*}{$\begin{array}{c}\text { Level } \\
\text { (g PSP/kg } \\
\text { diet) }\end{array}$} & \multirow{2}{*}{$\begin{array}{l}\text { Period } \\
\text { (day) }\end{array}$} & \multicolumn{5}{|c|}{ Growth performance } & \multicolumn{2}{|c|}{ Feed utilization } \\
\hline & & FW (g) & TWG (g) & ADG (g) & RGR (\%) & $\begin{array}{l}\text { SGR } \\
(\% / d)\end{array}$ & $\mathrm{FI}(\mathrm{g})$ & FCR \\
\hline 0 & \multirow{5}{*}{15} & $1.43^{\mathrm{E}}$ & $1.42^{\mathrm{E}}$ & $0.020^{\mathrm{E}}$ & $118.9^{\mathrm{E}}$ & $6.72^{\mathrm{E}}$ & $1.56^{\mathrm{D}}$ & $1.14^{\mathrm{ABC}}$ \\
\hline 2 & & $1.76^{\mathrm{DE}}$ & $1.75^{\mathrm{DE}}$ & $0.025^{\mathrm{DE}}$ & $147.1^{\mathrm{DE}}$ & $7.04^{\mathrm{DE}}$ & $2.53^{\mathrm{BCD}}$ & $1.46^{\mathrm{A}}$ \\
\hline 4 & & $2.87^{\mathrm{CD}}$ & $2.86^{\mathrm{CD}}$ & $0.040^{\mathrm{CD}}$ & $239.8^{\mathrm{CD}}$ & $7.71^{\mathrm{ABC}}$ & $2.24^{\mathrm{CD}}$ & $0.78^{\mathrm{BC}}$ \\
\hline 6 & & $4.29^{\mathrm{AB}}$ & $4.28^{\mathrm{AB}}$ & $0.060^{\mathrm{AB}}$ & $359.3^{\mathrm{AB}}$ & $8.29^{\mathrm{AB}}$ & $3.01^{\mathrm{ABC}}$ & $0.71^{\mathrm{C}}$ \\
\hline 8 & & $3.03^{\mathrm{BCD}}$ & $3.02^{\mathrm{BCD}}$ & $0.042^{\mathrm{BCD}}$ & $253.1 \mathrm{~B}^{\mathrm{CD}}$ & $7.80^{\mathrm{ABC}}$ & $2.67^{\mathrm{ABCD}}$ & $0.90^{\mathrm{BC}}$ \\
\hline 0 & \multirow{5}{*}{30} & $1.43^{\mathrm{E}}$ & $1.42^{\mathrm{E}}$ & $0.020^{\mathrm{E}}$ & $118.9^{\mathrm{E}}$ & $6.72^{\mathrm{E}}$ & $1.56^{\mathrm{D}}$ & $1.14^{\mathrm{ABC}}$ \\
\hline 2 & & $2.64^{\mathrm{CDE}}$ & $2.63^{\mathrm{CDE}}$ & $0.037^{\mathrm{CDE}}$ & $220.8^{\mathrm{CDE}}$ & $7.60^{\mathrm{CD}}$ & $2.28^{\mathrm{CD}}$ & $0.87^{\mathrm{BC}}$ \\
\hline 4 & & $3.49^{\mathrm{ABC}}$ & $3.48^{\mathrm{ABC}}$ & $0.049^{\mathrm{ABC}}$ & $291.8^{A B C}$ & $7.99^{\mathrm{ABC}}$ & $2.85^{\mathrm{ABC}}$ & $0.83^{\mathrm{BC}}$ \\
\hline 6 & & $3.41^{\mathrm{ABC}}$ & $3.40^{\mathrm{ABC}}$ & $0.048^{\mathrm{ABC}}$ & $285.4^{\mathrm{ABC}}$ & $7.95^{\mathrm{ABC}}$ & $2.66^{\mathrm{ABCD}}$ & $0.77^{\mathrm{BC}}$ \\
\hline 8 & & $2.76^{\mathrm{CD}}$ & $2.75^{\mathrm{CD}}$ & $0.038^{\mathrm{CD}}$ & $230.4^{\mathrm{CD}}$ & $7.65^{\mathrm{ABC}}$ & $3.57^{\mathrm{AB}}$ & $1.25^{\mathrm{AB}}$ \\
\hline 0 & \multirow{5}{*}{45} & $1.43^{\mathrm{E}}$ & $1.42^{\mathrm{E}}$ & $0.020^{\mathrm{E}}$ & $1118.9^{\mathrm{E}}$ & $6.72^{\mathrm{E}}$ & $1.56^{\mathrm{D}}$ & $1.14^{\mathrm{ABC}}$ \\
\hline 2 & & $3.01^{C D}$ & $3.00^{\mathrm{CD}}$ & $0.042^{\mathrm{CD}}$ & $251.9^{\mathrm{CD}}$ & $7.78^{\mathrm{ABC}}$ & $2.61^{\mathrm{ABCD}}$ & $0.88^{\mathrm{BC}}$ \\
\hline 4 & & $2.91^{\mathrm{CD}}$ & $2.89^{\mathrm{CD}}$ & $0.040^{\mathrm{CD}}$ & $242.9^{C D}$ & $7.72^{\mathrm{ABC}}$ & $2.62^{\mathrm{ABCD}}$ & $0.95^{\mathrm{BC}}$ \\
\hline 6 & & $3.66^{\mathrm{ABC}}$ & $3.65^{\mathrm{ABC}}$ & $0.051^{\mathrm{BC}}$ & $306.3^{B C}$ & $8.04^{\mathrm{ABC}}$ & $3.37^{\mathrm{ABC}}$ & $0.96^{\mathrm{BC}}$ \\
\hline 8 & & $2.92^{\mathrm{CD}}$ & $2.91^{\mathrm{CD}}$ & $0.041^{\mathrm{CD}}$ & $244.1^{C D}$ & $7.75^{\mathrm{ABC}}$ & $2.72^{\mathrm{ABCD}}$ & $0.94^{\mathrm{BC}}$ \\
\hline 0 & \multirow{5}{*}{60} & $1.43^{\mathrm{E}}$ & $1.42^{\mathrm{E}}$ & $0.020^{\mathrm{E}}$ & $1118.9^{\mathrm{E}}$ & $6.72^{\mathrm{E}}$ & $1.56^{\mathrm{D}}$ & $1.14^{\mathrm{ABC}}$ \\
\hline 2 & & $2.72^{\mathrm{CD}}$ & $2.70^{\mathrm{CD}}$ & $0.038^{\mathrm{CDE}}$ & $227.0^{\mathrm{CD}}$ & $7.65^{\mathrm{ABC}}$ & $2.61^{\mathrm{ABCD}}$ & $0.97^{\mathrm{ABC}}$ \\
\hline 4 & & $4.43^{\mathrm{A}}$ & $4.42^{\mathrm{A}}$ & $0.062^{\mathrm{A}}$ & $370.7^{A}$ & $8.33^{A}$ & $3.86^{\mathrm{A}}$ & $0.87^{\mathrm{BC}}$ \\
\hline 6 & & $2.92^{\mathrm{CD}}$ & $2.91^{\mathrm{CD}}$ & $0.040^{\mathrm{CD}}$ & $243.9^{\mathrm{CD}}$ & $7.71^{\mathrm{ABC}}$ & $2.81^{\mathrm{ABCD}}$ & $0.97^{\mathrm{ABC}}$ \\
\hline 8 & & $3.64^{\mathrm{ABC}}$ & $3.62^{\mathrm{ABC}}$ & $0.051^{\mathrm{ABC}}$ & $304.1^{\mathrm{ABC}}$ & $8.04^{\mathrm{ABC}}$ & $3.29^{\mathrm{ABC}}$ & $0.92^{\mathrm{BC}}$ \\
\hline \multicolumn{2}{|l|}{ \pm SE } & 0.236 & 0.236 & 0.003 & 19.84 & 0.119 & 0.238 & 0.092 \\
\hline \multicolumn{2}{|l|}{ P- value } & 0.0001 & 0.0001 & 0.0001 & 0.0001 & 0.0002 & 0.007 & 0.0008 \\
\hline
\end{tabular}

Means in the same column having different capital letters are significantly different (P $\leq$ 0.01).

\subsection{Whole fish composition:}

Carcass composition of Nile tilapia fry at the start and end of the experimental period is shown in Table 5. Generally, proximate analysis of the whole fish body at the start revealed higher crude protein (CP) and ash than at the end of the experiment, but dry matter (DM), ether extract (EE) and energy content $(E C)$ were lower at start than at the end of the experiment. At different periods, results indicated significantly $(P \leq 0.01)$ increased $D M, C P$ and ash content in the fry treated by PSP for 45 day compared with the other periods, but EE and EC content significantly $(P \leq 0.01)$ increased in fry fed PSP for 15 day compared with other periods.

The results of different levels of PSP showed significantly $(P \leq 0.01)$ improved DM and CP with increasing levels of PSP, where $\mathrm{L}_{4}(8 \mathrm{~g} \mathrm{PSP} / \mathrm{kg}$ diet) recorded the highest values of $\mathrm{DM}$ and $\mathrm{CP}$ compared with the other levels. Meanwhile, the control group $L_{0}(0 \mathrm{~g} \mathrm{PSP} / \mathrm{kg}$ diet $)$ gave the highest significantly $(P \leq 0.01)$ values of $E E$ and $E C$, but $L_{2}(4 \mathrm{~g} P S P / \mathrm{kg}$ diet $)$ recorded $(P \leq 0.01)$ highest ash content among all treatments.

The interaction effect between different levels of PSP with different periods on composition of Nile tilapia fry was given in Table 6 . Results revealed that the fry fed $8 \mathrm{~g} \mathrm{PSP} / \mathrm{kg}$ diet for 15 day; $6 \mathrm{~g} \mathrm{PSP} / \mathrm{kg}$ diet for 30,45 
day and $4 \mathrm{~g}$ PSP $/ \mathrm{kg}$ diet for 60 day were gave the high $(P \leq 0.01)$ values of $\mathrm{CP}$ compared with other treatments. Also, fry fed $6 \mathrm{~g} \mathrm{PSP} / \mathrm{kg}$ diet for 45 day recorded the highest $(P \leq 0.01)$ value of $D M$ among all treatments. While, the highest $(P \leq 0.01)$ value of EE was detected in fry fed $2 \mathrm{~g} \mathrm{PSP} / \mathrm{kg}$ diet for 15 day. Meanwhile, the control treatment $(0 \mathrm{~g} \mathrm{PSP} / \mathrm{kg}$ diet $)$ during all periods recorded the highest $(P \leq 0.01)$ values of $E C$ compared with other treatments.

2. The second period (the rearing period):

\subsection{Growth performance and feed utilization:}

Results in Table 7 showed the effect of different periods and levels of PSP on growth performance and feed utilization (FI and FCR) parameters of Nile tilapia fingerlings. All growth performance parameters and feed utilization ( $F I$ and $F C R$ ) were gradually $(P \leq 0.01)$ improved with increasing periods length until 45 day, then decreased with increasing the period till (60 day). Also, it could be noticed that the highest $(P \leq 0.01)$ values of all growth performance and feed utilization parameters compared with other periods was recorded at 45 day period.

In relation to the different levels of PSP, the results indicated that the FW, TWG, ADG and FI were significantly increased $(P \leq 0.01)$ with increasing levels of PSP, whereas $\mathrm{L}_{4}$ (8 g PSP/kg diet) reordered the highest values of FW, TWG and ADG; however, there was no significance between other levels of PSP ( $\mathrm{L}_{2}$ and $\mathrm{L}_{3}$ or 4 and $6 \mathrm{~g} / \mathrm{kg}$ diet, respectively). On the other hand, the RGR and $S G R$ significantly decreased $(P \leq 0.01)$ with increasing levels of PSP, where $L_{1}(2 \mathrm{~g} \mathrm{PSP} / \mathrm{kg}$ diet $)$ recorded the highest $(P \leq 0.01)$ values of RGR and SGR among all levels. While, the results indicated no significant differences in the FCR among different levels.

Table 5: Effects of different periods (day) and levels of PSP (g / kg diet) on the composition of Nile tilapia fry

\begin{tabular}{|c|c|c|c|c|c|}
\hline \multirow[b]{2}{*}{ Treat. } & \multirow[b]{2}{*}{ DM (\%) } & \multicolumn{4}{|c|}{$\%$ On dry matter basis } \\
\hline & & CP & EE & Ash & $\begin{array}{c}\text { EC } \\
(\mathrm{Kcal} / 100 \mathrm{~g})\end{array}$ \\
\hline \multicolumn{6}{|c|}{ At the start of the experiment } \\
\hline & 1.67 & 62.76 & 18.27 & 18.97 & 526.4 \\
\hline \multicolumn{6}{|c|}{ At the end of the experiment } \\
\hline \multicolumn{6}{|c|}{ Period (day) } \\
\hline $\mathbf{P}_{15}$ & $16.42^{\mathrm{B}}$ & $58.98^{\mathrm{B}}$ & $25.97^{A}$ & $15.06^{\mathrm{B}}$ & $577.7^{A}$ \\
\hline $\mathbf{P}_{30}$ & $16.76^{\mathrm{A}}$ & $59.61^{A}$ & $25.27^{\mathrm{C}}$ & $15.12^{\mathrm{B}}$ & $574.8^{\mathrm{B}}$ \\
\hline $\mathbf{P}_{45}$ & $16.66^{\mathrm{A}}$ & $59.91^{A}$ & $24.62^{B}$ & $15.47^{\mathrm{A}}$ & $570.3^{C}$ \\
\hline $\mathbf{P}_{60}$ & $16.06^{\mathrm{C}}$ & $59.26^{\mathrm{B}}$ & $25.35^{\mathrm{B}}$ & $15.39^{A}$ & $573.5^{\mathrm{B}}$ \\
\hline$\pm \mathrm{SE}$ & 0.042 & 0.122 & 0.124 & 0.057 & 0.634 \\
\hline P-value & 0.0001 & 0.0001 & 0.0001 & 0.0001 & 0.0001 \\
\hline \multicolumn{6}{|c|}{ Level (g PSP/ kg diet) } \\
\hline$L_{0}$ & $15.10^{\mathrm{E}}$ & $59.92^{\mathrm{AB}}$ & $26.43^{A}$ & $13.65^{\mathrm{D}}$ & $587.4^{\mathrm{A}}$ \\
\hline$L_{1}$ & $16.06^{\mathrm{D}}$ & $58.21^{\mathrm{D}}$ & $26.39^{A}$ & $15.41^{c}$ & $577.4^{\mathrm{B}}$ \\
\hline$L_{2}$ & $15.89^{\mathrm{C}}$ & $59.09^{c}$ & $24.75^{\mathrm{B}}$ & $16.17^{A}$ & $566.9^{D}$ \\
\hline$L_{3}$ & $17.28^{\mathrm{B}}$ & $59.71^{\mathrm{B}}$ & $24.66^{\mathrm{BC}}$ & $15.63^{B}$ & $569.6^{C}$ \\
\hline $\mathrm{L}_{4}$ & $18.06^{A}$ & $60.27^{A}$ & $24.29^{C}$ & $15.44^{\mathrm{C}}$ & $569.2^{C}$ \\
\hline \pm SE & 0.047 & 0.136 & 0.138 & 0.064 & 0.709 \\
\hline P- value & 0.0001 & 0.0001 & 0.0001 & 0.0001 & 0.0001 \\
\hline
\end{tabular}

Means in the same column having different capital letters are significantly different $(P \leq$ 0.01 ). 
Table 6: The interaction effect between different levels of PSP (g/kg diet) with different periods (day) on composition of Nile tilapia fry

\begin{tabular}{|c|c|c|c|c|c|c|}
\hline \multirow{2}{*}{$\begin{array}{c}\text { Level } \\
\text { (g PSP/kg diet) }\end{array}$} & \multirow{2}{*}{$\begin{array}{l}\text { Period } \\
\text { (day) }\end{array}$} & \multirow[b]{2}{*}{$\begin{array}{l}\text { DM } \\
(\%)\end{array}$} & \multicolumn{4}{|c|}{$\%$ On dry matter basis } \\
\hline & & & CP & EE & Ash & $\begin{array}{c}E C \\
(\mathrm{Kcal} / 100 \mathrm{~g})\end{array}$ \\
\hline 0 & \multirow{5}{*}{15} & $15.10^{1}$ & $59.92^{\mathrm{ABC}}$ & $26.43^{\mathrm{B}}$ & $13.65^{\mathrm{G}}$ & $587.4^{\mathrm{A}}$ \\
\hline 2 & & $16.36^{\mathrm{EF}}$ & $56.59^{\mathrm{F}}$ & $28.08^{A}$ & $15.32^{\mathrm{CDEF}}$ & $584.3^{A}$ \\
\hline 4 & & $15.58^{\mathrm{HI}}$ & $58.33^{\mathrm{DE}}$ & $25.72^{\mathrm{BC}}$ & $15.94^{\mathrm{ABC}}$ & $571.8^{\text {CDEF }}$ \\
\hline 6 & & $16.67^{\mathrm{E}}$ & $59.04^{\mathrm{BCDE}}$ & $25.76^{\mathrm{BC}}$ & $15.20^{\mathrm{DEF}}$ & $576.2^{\mathrm{BC}}$ \\
\hline 8 & & $18.39^{\mathrm{C}}$ & $61.00^{A}$ & $23.83^{\mathrm{EFGH}}$ & $15.17^{\mathrm{DEF}}$ & $569.0^{\text {CDEFG }}$ \\
\hline 0 & \multirow{5}{*}{30} & $15.10^{1}$ & $59.92^{\mathrm{ABC}}$ & $26.43^{\mathrm{B}}$ & $13.65^{\mathrm{G}}$ & $587.4^{\mathrm{A}}$ \\
\hline 2 & & $15.91^{\mathrm{FGH}}$ & $58.67^{\mathrm{CDE}}$ & $26.57^{\mathrm{B}}$ & $14.75^{\mathrm{F}}$ & $581.8^{\mathrm{AB}}$ \\
\hline 4 & & $16.25^{\mathrm{EFG}}$ & $58.27^{\mathrm{DE}}$ & $25.47^{\mathrm{BCD}}$ & $16.26^{\mathrm{A}}$ & $569.1^{\text {CDEFG }}$ \\
\hline 6 & & $17.58^{\mathrm{D}}$ & $60.89^{A}$ & $23.72^{\mathrm{FGH}}$ & $15.39^{\mathrm{CDEF}}$ & $567.3^{\mathrm{EFG}}$ \\
\hline 8 & & $18.97^{\mathrm{AB}}$ & $60.30^{A B}$ & $24.17^{\text {DEFGH }}$ & $15.53^{\mathrm{BCDE}}$ & $568.3^{\text {DEFG }}$ \\
\hline 0 & \multirow{5}{*}{45} & $15.10^{1}$ & $59.92^{\mathrm{ABC}}$ & $26.43^{\mathrm{B}}$ & $13.65^{\mathrm{G}}$ & $587.4^{\mathrm{A}}$ \\
\hline 2 & & $16.66^{\mathrm{E}}$ & $59.86^{\mathrm{ABC}}$ & $24.45^{\text {CDEFG }}$ & $15.69^{\mathrm{ABCDE}}$ & $568.4^{\text {DEFG }}$ \\
\hline 4 & & $15.90^{\mathrm{FGH}}$ & $58.96^{\mathrm{BCDE}}$ & $24.93^{\mathrm{CDEF}}$ & $16.11^{\mathrm{AB}}$ & $567.9^{\text {DEFG }}$ \\
\hline 6 & & $19.46^{\mathrm{A}}$ & $60.64^{A}$ & $23.41^{\mathrm{GH}}$ & $15.95^{\mathrm{ABC}}$ & $563.0^{\mathrm{GH}}$ \\
\hline 8 & & $16.20^{\mathrm{EFG}}$ & $60.19^{\mathrm{AB}}$ & $23.89^{\mathrm{EFGH}}$ & $15.93^{\mathrm{ABC}}$ & $564.9 F^{\mathrm{GH}}$ \\
\hline 0 & \multirow{5}{*}{60} & $1015.10^{\prime}$ & $59.92^{\text {ABC }}$ & $26.43^{\mathrm{B}}$ & $13.65^{\mathrm{G}}$ & $587.4^{\mathrm{A}}$ \\
\hline 2 & & $15.31^{1}$ & $57.71^{\mathrm{EF}}$ & $26.44^{\mathrm{B}}$ & $15.85^{\mathrm{ABCD}}$ & $575.0^{\mathrm{BCD}}$ \\
\hline 4 & & $15.83^{\mathrm{GH}}$ & $60.79^{A}$ & $22.86^{\mathrm{H}}$ & $16.35^{A}$ & $558.7^{\mathrm{H}}$ \\
\hline 6 & & $15.41^{\mathrm{HI}}$ & $58.26^{\mathrm{DE}}$ & $25.76^{\mathrm{BC}}$ & $15.98^{\mathrm{ABC}}$ & $571.7^{\mathrm{CDEF}}$ \\
\hline 8 & & $18.67^{\mathrm{BC}}$ & $59.61^{\mathrm{ABCD}}$ & $25.27^{\mathrm{BCDE}}$ & $15.12^{\mathrm{EF}}$ & $574.8^{\mathrm{BCDE}}$ \\
\hline \multicolumn{2}{|l|}{$\pm \mathrm{SE}$} & 0.094 & 0.273 & 0.277 & 0.128 & 1.419 \\
\hline \multicolumn{2}{|l|}{ P-value } & 0.0001 & 0.0001 & 0.0001 & 0.0001 & 0.0001 \\
\hline
\end{tabular}

Means in the same column having different capital letters are significantly different (P $\leq 0.01)$.

Table 7: Effect of different periods (day) and levels of PSP ( $\mathrm{g} / \mathrm{kg}$ diet) on growth performance and feed efficiency parameters of Nile tilapia fingerlings during the rearing period

\begin{tabular}{|c|c|c|c|c|c|c|c|}
\hline \multirow{2}{*}{ Treat. } & \multicolumn{5}{|c|}{ Growth performance } & \multicolumn{2}{|c|}{ Feed utilization } \\
\hline & $\mathbf{F W}(\mathrm{g})$ & $\overline{\text { TWG }(\mathrm{g})}$ & $\overline{\text { ADG }(g)}$ & RGR (\%) & SGR $(\% / d)$ & FI (g) & FCR \\
\hline \multicolumn{8}{|c|}{ Period (day) } \\
\hline$P_{15}$ & $24.26^{\mathrm{B}}$ & $20.23^{\mathrm{B}}$ & $0.241^{\mathrm{B}}$ & $515.19^{\mathrm{B}}$ & $2.15^{\mathrm{B}}$ & $48.91^{\mathrm{C}}$ & $2.45^{A}$ \\
\hline$\widetilde{P_{30}}$ & $25.81^{\mathrm{B}}$ & $21.25^{\mathrm{B}}$ & $0.253^{\mathrm{B}}$ & $470.94^{\mathrm{B}}$ & $2.06^{\mathrm{B}}$ & $52.65^{\mathrm{B}}$ & $2.51^{A}$ \\
\hline $\mathbf{P}_{45}$ & $30.57^{\mathrm{A}}$ & $26.38^{A}$ & $0.314^{\mathrm{A}}$ & $624.77^{A}$ & $2.35^{\mathrm{A}}$ & $56.11^{\mathrm{A}}$ & $2.18^{\mathrm{B}}$ \\
\hline$\overline{P_{60}}$ & $25.73^{B}$ & $21.17^{\mathrm{B}}$ & $0.252^{\mathrm{B}}$ & $481.42^{\mathrm{B}}$ & $2.08^{\mathrm{B}}$ & $53.81^{\mathrm{AB}}$ & $2.55^{A}$ \\
\hline \pm SE & 0.731 & 0.731 & 0.008 & 17.68 & 0.033 & 0.806 & 0.089 \\
\hline P-value & 0.0001 & 0.0001 & 0.0001 & 0.0001 & 0.0001 & 0.0001 & 0.028 \\
\hline \multicolumn{8}{|c|}{ Level (g PSP/ kg diet) } \\
\hline$L_{0}$ & $15.16^{\mathrm{C}}$ & $12.75^{\mathrm{C}}$ & $0.152^{\mathrm{C}}$ & $529.5^{\mathrm{B}}$ & $2.18^{\mathrm{AB}}$ & $30.40^{\mathrm{D}}$ & 2.43 \\
\hline $\mathrm{L}_{1}$ & $25.94^{\mathrm{B}}$ & $22.21^{\mathrm{B}}$ & $0.265^{\mathrm{B}}$ & $594.1^{A}$ & $2.28^{A}$ & $49.18^{C}$ & 2.30 \\
\hline$L_{2}$ & $29.67^{A}$ & $24.77^{A}$ & $0.295^{\mathrm{A}}$ & $514.4^{\mathrm{B}}$ & $2.15^{\mathrm{B}}$ & $59.81^{B}$ & 2.43 \\
\hline$L_{3}$ & $30.73^{A}$ & $25.47^{A}$ & $0.303^{A}$ & $484.2^{\mathrm{B}}$ & $2.09^{B}$ & $61.34^{\mathrm{AB}}$ & 2.46 \\
\hline $\mathrm{L}_{4}$ & $31.46^{\mathrm{A}}$ & $26.09^{A}$ & $0.311^{\mathrm{A}}$ & $493.0^{B}$ & $2.10^{B}$ & $63.61^{A}$ & 2.48 \\
\hline \pm SE & 0.817 & 0.817 & 0.009 & 19.77 & 0.038 & 0.902 & 0.100 \\
\hline P- value & 0.0001 & 0.0001 & 0.0001 & 0.002 & 0.005 & 0.0001 & 0.718 \\
\hline
\end{tabular}

Means in the same column having different capital letters are significantly different $(P \leq$ 0.01). 
Farrag, F. H. et al.

The interaction effect between different levels of PSP and different periods on growth performance of Nile tilapia fingerlings is presented in Table 8. The results indicated that treating with $6 \mathrm{~g} \mathrm{PSP} / \mathrm{kg}$ diet for 45 day showed the highest $(P \leq 0.01)$ values of FW, TWG and ADG compared with other treatments. While, the level $2 \mathrm{~g} \mathrm{PSP} / \mathrm{kg}$ diet for 45 day gave the highest $(\mathrm{P} \leq$ $0.01)$ RGR and SGR among all treatments. Also, the results indicated no significant $(P \geq 0.05)$ differences in $F C R$ among all treatments.

Table 8: Interaction effect between different levels of PSP ( $\mathrm{g} / \mathrm{kg}$ diet) with different periods (day) on growth performance of Nile tilapia fingerlings

\begin{tabular}{|c|c|c|c|c|c|c|c|c|}
\hline \begin{tabular}{|c|} 
Level \\
(g/kg diet)
\end{tabular} & $\begin{array}{c}\text { Period } \\
\text { (day) }\end{array}$ & FW (g) & TWG (g) & ADG (g) & RGR (\%) & SGR $(\% / d)$ & $\mathrm{FI}(\mathrm{g})$ & FCR \\
\hline 0 & \multirow{5}{*}{15} & $15.16^{\mathrm{F}}$ & $12.75^{\mathrm{G}}$ & $0.152^{\mathrm{F}}$ & $529.5^{\mathrm{BCD}}$ & $2.18^{\mathrm{ABCD}}$ & $30.40^{\prime}$ & 2.43 \\
\hline 2 & & $19.56^{\mathrm{EF}}$ & $16.67^{\mathrm{FG}}$ & $0.199^{\mathrm{EF}}$ & $577.4^{\mathrm{ABCD}}$ & $2.27^{\mathrm{ABC}}$ & $39.32^{\mathrm{HI}}$ & 2.39 \\
\hline 4 & & $27.62^{\mathrm{BCDE}}$ & $23.45^{\mathrm{ABCDEF}}$ & $0.279^{\mathrm{ABCDE}}$ & $563.3^{\mathrm{ABCD}}$ & $2.25^{\mathrm{ABCD}}$ & $56.22^{\mathrm{DEF}}$ & 2.43 \\
\hline 6 & & $32.54^{\mathrm{ABC}}$ & $26.66^{\mathrm{ABCD}}$ & $0.317^{\mathrm{ABC}}$ & $452.7^{\mathrm{BCD}}$ & $2.03^{\mathrm{BCD}}$ & $63.72^{\mathrm{BCDE}}$ & 2.42 \\
\hline 8 & & $26.42^{\mathrm{CDE}}$ & $21.64^{\mathrm{DEF}}$ & $0.257^{\mathrm{CDE}}$ & $453.1^{\mathrm{BCD}}$ & $2.03^{\mathrm{BCD}}$ & $54.87^{\mathrm{EF}}$ & 2.56 \\
\hline$\overline{0}$ & \multirow{5}{*}{30} & $1515.16^{\mathrm{F}}$ & $12.75^{\mathrm{G}}$ & $0.152^{\mathrm{F}}$ & $529.5^{\mathrm{BCD}}$ & $2.18^{\mathrm{ABCD}}$ & $30.40^{1}$ & 2.43 \\
\hline 2 & & $21.51^{\text {DEF }}$ & $17.49^{\mathrm{EGF}}$ & $0.208^{\mathrm{DEF}}$ & $436.0^{\mathrm{CD}}$ & $1.99^{\mathrm{CD}}$ & $44.90^{\mathrm{GH}}$ & 2.60 \\
\hline 4 & & $30.82^{\mathrm{ABC}}$ & $25.58^{\mathrm{ABCDE}}$ & $0.305^{\mathrm{ABCD}}$ & $488.3^{\mathrm{BCD}}$ & $2.11^{\mathrm{BCD}}$ & $59.32^{\mathrm{CDEF}}$ & 2.32 \\
\hline 6 & & $26.58^{\mathrm{CDE}}$ & $21.45^{\mathrm{DEFG}}$ & $0.255^{\mathrm{CDEF}}$ & $418.4^{\mathrm{CD}}$ & $1.94^{\mathrm{CD}}$ & $54.01^{\mathrm{FG}}$ & 2.59 \\
\hline 8 & & $34.98^{\mathrm{ABC}}$ & $28.97^{\mathrm{ABCD}}$ & $0.345^{\mathrm{ABC}}$ & $482.5^{\mathrm{BCD}}$ & $2.09^{\mathrm{BCD}}$ & $74.63^{A}$ & 2.59 \\
\hline$\overline{0}$ & \multirow{5}{*}{45} & $15.16^{\mathrm{F}}$ & $12.75^{\mathrm{G}}$ & $0.152^{\mathrm{F}}$ & $529.5^{\mathrm{BCD}}$ & $2.18^{\mathrm{ABCD}}$ & $30.40^{1}$ & 2.43 \\
\hline 2 & & $34.69^{\mathrm{ABC}}$ & $30.68^{\mathrm{ABC}}$ & $0.365^{\mathrm{AB}}$ & $765.4^{\mathrm{A}}$ & $2.57^{\mathrm{A}}$ & $58.19^{\mathrm{CDEF}}$ & 1.91 \\
\hline 4 & & $30.21^{\mathrm{ABCD}}$ & $25.82^{\mathrm{ABCDE}}$ & $0.307^{\mathrm{ABCD}}$ & $587.9^{\mathrm{ABC}}$ & $2.29^{\mathrm{ABC}}$ & $57.56^{\mathrm{CDEF}}$ & 2.25 \\
\hline 6 & & $36.68^{A}$ & $31.27^{\mathrm{AB}}$ & $0.372^{\mathrm{A}}$ & $577.7^{\mathrm{ABCD}}$ & $2.27^{\mathrm{ABC}}$ & $69.23^{\mathrm{AB}}$ & 2.24 \\
\hline 8 & & $36.12^{\mathrm{AB}}$ & $31.39^{\mathrm{A}}$ & $0.374^{\mathrm{A}}$ & $663.3^{\mathrm{AB}}$ & $2.42^{\mathrm{AB}}$ & $65.18^{\mathrm{ABCD}}$ & 2.08 \\
\hline$\overline{0}$ & \multirow{5}{*}{60} & $15.16^{\mathrm{F}}$ & $12.75^{\mathrm{G}}$ & $0.152^{\mathrm{F}}$ & $529.5^{\mathrm{BCD}}$ & $2.18^{A B C D}$ & $30.40^{1}$ & 2.43 \\
\hline 2 & & $28.00^{\mathrm{ABCDE}}$ & $23.99^{\mathrm{ABCDEF}}$ & $0.286^{\mathrm{ABCDE}}$ & $597.9^{\mathrm{ABC}}$ & $2.31^{\mathrm{ABC}}$ & $54.33^{\mathrm{EFG}}$ & 2.28 \\
\hline 4 & & $30.04^{\mathrm{ABCD}}$ & $24.24^{\mathrm{ABCDEF}}$ & $0.289^{\mathrm{ABCDE}}$ & $418.2^{\mathrm{CD}}$ & $1.96^{\mathrm{CD}}$ & $66.12^{\mathrm{ABC}}$ & 2.73 \\
\hline 6 & & $27.13^{\mathrm{CDE}}$ & $22.52^{\mathrm{BCDEF}}$ & $0.268^{\mathrm{BCDE}}$ & $488.1^{\mathrm{BCD}}$ & $2.11^{\mathrm{BCD}}$ & $58.41^{\mathrm{CDEF}}$ & 2.60 \\
\hline 8 & & $28.32^{\mathrm{ABCDE}}$ & $22.34^{\mathrm{CDEF}}$ & $0.266^{\mathrm{BCDE}}$ & $373.3^{\mathrm{D}}$ & $1.85^{\mathrm{D}}$ & $59.77^{\mathrm{BCDEF}}$ & 2.69 \\
\hline \multicolumn{2}{|l|}{$\pm \mathrm{SE}$} & 1.635 & & 39.54 & 0.076 & 39.54 & $\begin{array}{l}1.804 \\
\end{array}$ & 0.200 \\
\hline \multicolumn{2}{|l|}{ P- value } & 0.0001 & 0.004 & 0.004 & 0.0040 & 0.0041 & 0.0001 & 0.824 \\
\hline
\end{tabular}

Means in the same column having different capital letters are significantly different $(\mathbf{P} \leq$ 0.001).

\subsection{Whole fish composition:}

Proximate analysis of the whole body of Nile tilapia fingerlings at the end of the rearing period is given in Table 9. Regarding the different periods, the data indicated that no clear trend in all components of chemical analysis of the whole body, but fish fed with PSP for 30 day recorded the highest $(P \leq$ $0.01) \mathrm{DM}$ content than other periods. Although, CP content was decreased ( $P$ $\leq 0.01$ ) with increasing the periods; yet, the 15 day period gave the best value among all periods. In contrary, the EE and EC contents were increased $(P \leq$ 0.01 ) with increasing periods, the 45 day period had the highest values of $E E$ and EC compared with other periods. However, there was no significant $(P \geq$ $0.05)$ effect on ash content among all periods.

Concerning the different levels of PSP, the results showed that DM, $E E$, ash and $E C$ contents were increased $(P \leq 0.01)$ with increasing levels of 
PSP, whereas the $L_{3}(6 \mathrm{~g} \mathrm{PSP} / \mathrm{kg}$ diet) had the highest $(P \leq 0.01)$ values of $\mathrm{DM}$, EE and EC than other levels of PSP. $\mathrm{L}_{4}(8 \mathrm{~g} \mathrm{PSP} / \mathrm{kg}$ diet $)$ had the highest value $(P \leq 0.01)$ of ash among all levels of PSP. Contrariwise, CP content was decreased $(P \leq 0.01)$ with increasing levels of $P S P$, meanwhile $\mathrm{L}_{1}(2 \mathrm{~g} \mathrm{PSP} / \mathrm{kg}$ diet$)$ had the highest $(\mathrm{P} \leq 0.01)$ value of $\mathrm{CP}$ content among all levels of PSP.

Table 9: Effects of different periods and PSP levels on carcass composition of Nile tilapia fingerlings during the rearing period

\begin{tabular}{|l|c|c|c|c|c|}
\hline \multirow{2}{*}{ Treat. } & \multirow{2}{*}{ DM (\%) } & \multicolumn{5}{c|}{ \% On dry matter basis } \\
\cline { 3 - 6 } & & CP & EE & Ash & EC \\
(Kcal/100 g)
\end{tabular}

Means in the same column having different capital letters are significantly different (P $\leq$ 0.01).

The interaction effect between different levels of PSP and different periods on composition of Nile tilapia fingerlings is presented in Table 10. Results revealed that the fish fed with $6 \mathrm{~g} \mathrm{PSP} / \mathrm{kg}$ diet for 30 day had the best DM content. While, the levels $2 \mathrm{~g} \mathrm{PSP} / \mathrm{kg}$ diet for 60 day and $4 \mathrm{~g} \mathrm{PSP} / \mathrm{kg}$ diet for 15 day had the highest $(P \leq 0.01)$ values of $C P$ content compared with all treatments. On the other hand, the level of $6 \mathrm{~g} \mathrm{PSP} / \mathrm{kg}$ diet for 15 day gave the highest $(P \leq 0.01)$ values of EE and EC among all treatments. Also, the levels $8 \mathrm{~g} \mathrm{PSP} / \mathrm{kg}$ diet for 30 day and $4 \mathrm{~g} \mathrm{PSP} / \mathrm{kg}$ diet for 15 day led to the highest $(P \leq 0.01)$ values of ash content than all treatments. 
Farrag, F. H. et al.

Table 10: Interactions effect between different levels of PSP with different periods on carcass composition of Nile tilapia fingerlings during the rearing period.

\begin{tabular}{|c|c|c|c|c|c|c|}
\hline \multirow{2}{*}{$\begin{array}{c}\text { Level } \\
\text { (g/kg diet) }\end{array}$} & \multirow{2}{*}{$\begin{array}{c}\text { Period } \\
\text { (day) }\end{array}$} & \multirow[b]{2}{*}{ DM (\%) } & \multicolumn{4}{|c|}{$\%$ On Dry matter basis } \\
\hline & & & $\begin{array}{l}\mathbf{C P} \\
(\%)\end{array}$ & $\begin{array}{l}\text { EE } \\
(\%)\end{array}$ & $\begin{array}{c}\text { Ash } \\
(\%)\end{array}$ & $\begin{array}{c}\text { EC } \\
(\text { Kcal/100 g) }\end{array}$ \\
\hline 0 & \multirow{5}{*}{15} & $23.80^{\mathrm{F}}$ & $60.05^{\mathrm{CDE}}$ & $26.54^{\mathrm{EF}}$ & $13.41^{\mathrm{D}}$ & $316.20^{\mathrm{EF}}$ \\
\hline 2 & & $25.72^{C}$ & $61.87^{\mathrm{B}}$ & $23.79^{\mathrm{G}}$ & $14.34^{\mathrm{ABCD}}$ & $292.07^{\mathrm{G}}$ \\
\hline 4 & & $25.34^{\mathrm{CD}}$ & $64.22^{A}$ & $21.07^{\mathrm{H}}$ & $14.71^{\mathrm{A}}$ & $268.77^{\mathrm{H}}$ \\
\hline 6 & & $25.98^{\mathrm{C}}$ & $56.75^{\mathrm{GH}}$ & $29.80^{A}$ & $13.40^{\mathrm{D}}$ & $343.67^{A}$ \\
\hline 8 & & $22.81^{\mathrm{G}}$ & $60.26^{\mathrm{BCD}}$ & $25.33^{\mathrm{FG}}$ & $14.63^{\mathrm{AB}}$ & $304.97^{\mathrm{FG}}$ \\
\hline 0 & \multirow{5}{*}{30} & $23.80^{\mathrm{F}}$ & $60.05^{\mathrm{CDE}}$ & $26.54^{\mathrm{EF}}$ & $13.41^{\mathrm{D}}$ & $316.20^{\mathrm{EF}}$ \\
\hline 2 & & $23.94^{\mathrm{EF}}$ & $59.24^{\text {DEF }}$ & $26.45^{\mathrm{EF}}$ & $14.32^{\mathrm{ABCD}}$ & $314.57^{\mathrm{EF}}$ \\
\hline 4 & & $24.65^{\mathrm{DE}}$ & $61.63^{\mathrm{BC}}$ & $24.42^{\mathrm{G}}$ & $13.94^{\mathrm{ABCD}}$ & $297.83^{G}$ \\
\hline 6 & & $28.74^{A}$ & $59.16^{\text {DEF }}$ & $26.97^{\mathrm{DE}}$ & $13.87^{\mathrm{ABCD}}$ & $319.37^{\mathrm{DE}}$ \\
\hline 8 & & $27.64^{\mathrm{B}}$ & $58.28^{\mathrm{EFG}}$ & $26.98^{\mathrm{DE}}$ & $14.75^{\mathrm{A}}$ & $318.57^{\mathrm{DE}}$ \\
\hline 0 & \multirow{5}{*}{45} & $23.80^{\mathrm{F}}$ & $60.05^{\mathrm{CDE}}$ & $26.54^{\mathrm{EF}}$ & $13.41^{\mathrm{D}}$ & $316.20^{\mathrm{EF}}$ \\
\hline 2 & & $24.63^{\mathrm{DEF}}$ & $59.36^{\mathrm{DEF}}$ & $26.99^{\mathrm{DE}}$ & $13.65^{\mathrm{BCD}}$ & $319.77^{\mathrm{DE}}$ \\
\hline 4 & & $25.81^{\mathrm{C}}$ & $58.13^{\mathrm{FG}}$ & $28.32^{\mathrm{ABCD}}$ & $13.55^{\mathrm{CD}}$ & $331.13^{\mathrm{ABCD}}$ \\
\hline 6 & & $25.71^{\mathrm{C}}$ & $58.00^{\mathrm{FGH}}$ & $27.54^{\mathrm{CDE}}$ & $14.46^{\mathrm{ABC}}$ & $323.63^{\mathrm{CDE}}$ \\
\hline 8 & & $25.36^{\mathrm{CD}}$ & $58.38^{\mathrm{EFG}}$ & $27.01^{\mathrm{DE}}$ & $14.61^{\mathrm{AB}}$ & $319.00^{\mathrm{DE}}$ \\
\hline 0 & \multirow{5}{*}{60} & $23.80^{\mathrm{F}}$ & $60.05^{\mathrm{CDE}}$ & $26.54^{\mathrm{EF}}$ & $13.41^{\mathrm{D}}$ & $316.20^{\mathrm{EF}}$ \\
\hline 2 & & $20.74^{\mathrm{H}}$ & $64.73^{A}$ & $20.75^{\mathrm{H}}$ & $14.52^{\mathrm{ABC}}$ & $266.27^{\mathrm{H}}$ \\
\hline 4 & & $26.90^{\mathrm{B}}$ & $56.27^{\mathrm{H}}$ & $29.75^{\mathrm{AB}}$ & $13.98^{\mathrm{ABCD}}$ & $342.77^{\mathrm{AB}}$ \\
\hline 6 & & $27.06^{\mathrm{B}}$ & $56.90^{\mathrm{GH}}$ & $28.82^{\mathrm{ABC}}$ & $14.28^{\mathrm{ABCD}}$ & $334.63^{\mathrm{ABC}}$ \\
\hline 8 & & $26.00^{C}$ & $57.60^{\mathrm{FGH}}$ & $28.25^{\mathrm{BCD}}$ & $14.15^{\mathrm{ABCD}}$ & $329.93^{\mathrm{BCD}}$ \\
\hline \multicolumn{2}{|l|}{$\pm \mathrm{SE}$} & 0.155 & 0.332 & 0.287 & 0.182 & 2.446 \\
\hline \multicolumn{2}{|l|}{ P-value } & 0.0001 & 0.0001 & 0.0001 & 0.0001 & 0.0001 \\
\hline
\end{tabular}

Means in the same column having different capital letters are significantly different (P $\leq$ 0.01).

\section{DISSCUSION}

The previous results related to the effect of using feed additives in fish draw the attention to examine many of chemicals as well as medicinal herbs and plants on the basis of favorable and economical reflected results. Nowadays, most of the chemical materials are out of use because of health problems. In the same time, tremendous knowledge was concentrated to the organic materials. However, the results did not show exact positive or negative trend in most cases. No-doubt, the obtained results are related to a lot of factors. In this trend, the present results showed positive effect of pawpaw seeds powder (PSP) in diet of Nile tilapia. These results did not agree with some of previous studies. In this trend, it was found that addition of PSP caused decreased of growth performance of $O$. niloticus during 30 day treatment period (Ekanem and Okoronkwo, 2003 and Abbas and Abbas, 2011). Also, using dietary Aloe vera latex did not show significant differences in growth performance parameters and feed conversion ratio (Jegede, 2009). In other study, Jegede (2010) reported that the best overall growth response was obtained in Nile tilapia fed the basal diet; on the other side the weight 
gain, average daily gain and specific growth rate were poor in fish fed the Hibiscus Rosa-sinensis leaf meal diets.

So, the contrary trend to other studies, as well as the convulsions may be related to the different age of fish (stages), species, level of PSP and duration of exposure. Where in the present study, tilapia fish fry was fed after hatching and yolk sac absorption by different levels of PSP $(0,2,4,6$ and 8 $\mathrm{g} / \mathrm{kg}$ diet) for different periods (15, 30, 45 and 60 days). While, the contrary were used in other studies, adult male tilapia with an average weight $40 \mathrm{~g}$ were fed with different levels of PSP (low dose of $4.9 \mathrm{~g} / \mathrm{kg} /$ day or high dose of $9.8 \mathrm{~g} / \mathrm{kg} /$ day) for 30 day only (Ekanem and Okoronkwo, 2003 and Abbas and Abbas, 2011). Similarly, Jegede and Fagbenro (2008) used different levels of PSP $(0,0.5,1.0,1.5$ and $2 \mathrm{~g} / \mathrm{kg}$ diet) for 60 day only for adult Nile tilapia with an average weight of $40 \mathrm{~g}$. Generally, in addition to the effect of level and period of administration of ripe pawpaw seed (C. papaya), its extract induced variable responses according to the kind of extract, dose, duration and mode of administration (Lohiya et al., 2002).

In addition, the improvement in growth performance and feed utilization of Nile tilapia in the present study may be due to the PSP content of some useful life fauna with bacteriostatic effects against several enteropathogens such as Bacillus subtilis, Enterobacter cloacae, Escherichia coli, Salmonella typhi, Staphylococcus aureus, Proteus vulgaris, Pseudomonas aeruginosa and Klebsiella pneumoniae (Osato et al., 1993). Moreover, the seeds of irrespective stage of fruit maturity have bacteriostatic activity on gram positive and negative organisms. The papaya seed macerate has a clinical potential on conjugal $\mathrm{R}$ plasmid transfer from Salmonella typhimurium to $E$. coli, in vitro and in the digestive tract of genotobiotic mice (Leite et al., 2005). Furthermore, the aqueous seed extract of Carica papaya contains a number of bioactive compounds (benzyisothiocyanate, alkaloids, flavinoids), which have biological activities, e.g. anthelmintic (Kermanshai et al., 2001), antimicrobial, antitumor (Banerjee, 2002), and antiparasitic (HounzangbeAdote et al., 2005).

In present study, the results of various survival rates (SR) of $O$. niloticus fry were concluded during the feeding period with PSP in different periods, where SR of fish after 15 day was in range from 86.66 to $96.67 \%$, after 30 day was $80.00-88.33 \%$, after 45 day was 75.00 to $85.00 \%$ and after 60 day was 73.33 to $85.00 \%$. While, the overall mean of SR ranged from 77.66 to $84.00 \%$ during the different periods, as well as, the overall mean of SR was in range from 75.00 to $87.50 \%$ due to the different levels of PSP. The previous obtained percentage of SR of Nile tilapia fry was in normal 70 to $80 \%$ (Popma and Lovshin, 1995).

Ayotunde and Ofem (2008) reported that use of PSP at level of 9.8 $\mathrm{g} / \mathrm{kg}$ of fish/day indiscriminately in their farm to control prolific breeding of tilapia and it has resulted in high and regular death of fry, fingerling and adult tilapia in pond. They attributed the high death of fry and fingerlings to the PSP contents of toxic substance called carpine (12, 25-diazatricyclo [22.2.2.211, 14] triacontane-3, 16-dione). In the contrary, the high SR \% in the present study may be due to low levels used of PSP, and at different periods to 
Farrag, F. H. et al.

determine the safety level during the best exposure period. Also, it is important to mention that the aqueous seed extract of $C$. papaya contains a number of bioactive compounds such as benzyisothiocyanate, alkaloids and flavinoids (Kermanshai et al., 2001), which may save the SR of Nile tilapia fry treated with PSP within the normal range (70 to $80 \%$ ) during this critical stage (Popma and Lovshin, 1995).

Growth and feed conversion traits reflect the body composition of the fish. In the same time, species, genetic strain, sex and stage of reproductive cycle lead to different nutritional requirements and thereby body composition (Jauncey, 1998). The present results indicated that treated with high dose of PSP $(8 \mathrm{~g} / \mathrm{kg}$ diets) in the treated period lead to increase dry matter (DM) and crude protein (CP) in the whole body and decreased ether extract (EE). In contrary, fish treated with high dose of PSP during the rearing period decreased DM and CP, as well as increased of $E E$ in the whole body of adult Nile tilapia. The results of rearing period are in agreement with Abbas and Abbas (2011). They found that Nile tilapia treated with high dose of PSP (6 $\mathrm{g} / \mathrm{kg} /$ day) exhibited lowest meat quality, reflected in lower DM and CP, as well as decrease of total muscle protein and total lipids and increase in water and EE contents (Niragh and Simmone, 1984). Increasing water content in muscle of fish treated with PSP especially with high dose was explained by Weatherly and Gills (1987), they concluded that depletion of body constituents (protein and lipids) results in tissue hydration of inverse dynamic relationships with protein, as well as lipids and water content in the muscles.

Generally, the opposite results regarding the fish body composition between the experimental periods ( $1^{\text {st }}$ and $2^{\text {nd }}$ periods) may be related to fish age (fry, in feeding period or fingerlings in rearing period), as well as nutritional and physiological status of fish. It seems that to achieve improvement in most of growth performance parameters, survival, FCR and fish composition, pawpaw seeds powder (PSP) could be added to the diet very early after hatching on one side and the level of pawpaw seeds powder could be raised to $6 \mathrm{~g} / \mathrm{kg}$ diet for a long period (45 day) on the other side. The present study showed also the need for further research work on the PSP contents of toxic substances (carpine).

\section{REFERENCES}

Abbas, H. H. and W. T. Abbas (2011). Assessment study on the use of pawpaw; Carica papaya seeds to control Oreochromis niloticus breeding. Pakistan Journal of Biological Sciences, 14: 1117-1123.

Abdelhamid, A. M. (2000). Scientific Fundamentals of Fish Production and Husbandry. $2^{\text {nd }}$. Ed., Mansoura Faculty of Agriculture. (ISBN: 9775526-04-1).

AOAC., (2000). Association of Official Analytical Chemists of Official Methods of Analysis, $17^{\text {th }}$ Ed. Washington, DC. 
Ayotunde, E. O. and B.O. Ofem (2008). Acute and chronic toxicity of pawpaw (Carica papaya) seed powder to Nile tilapia Oreochromis niloticus (Linne 1757), fingerlings. African Journal of Biotechnology, 7 (13): 2265-2274.

Banerjee, J. (2002). Tissue culture and transformation studies in Indian cultivars of papaya (Carica papaya L.) Ph D thesis, Plant tissue culture division, National chemical laboratory pune, India; p: 26- 27.

Baruah, K.; P. Norouzitallab; D. Debnath; A .K. Pal and N.P. Sahu (2008). Organic acids as non-antibiotic nutraceuticals in fish and prawn feed. Aquacult. Health Internat.12: 4-6.

Bolu, S.A.O.; F. E. Sola-Ojo; O.A. Olorunsanya and K. Idris (2009). Effect of graded levels of dried pawpaw (Carica papaya) seed on the performance, haematology, serum biochemistry and carcass evaluation of chicken Broilers. International Journal of Poultry Science, 8 (9): 905-909.

Casey, R. D. C. (1960). Alleged antifertility plants of India. Indian J. Med. Sci. $14,590-600$.

Chinoy, N.j.; K.G. Patel and C. Sunita (1997). Reversible effects of aqueous extract of papaya seed on microenvironment and sperm metabolism of caudal epididymis of rat. J. Medicinal and Aromatic Plant Sci., 19: 717723.

Duncan, D.B. (1955). Multiple ranges and multiple F-tests. Biometrics, 11:142.

Ekanem, S.B. and P.O. Bassey (2003). Effect of pawpaw seed (Carica papaya) as antifertility agent in female Nile tilapia (Oreochromis niloticus). Journal of Aquaculture in the Tropics, 18 (2): 181-188.

Ekanem, S.B. and T.E. Okoronkwo (2003). Pawpaw seed as fertility control agent on male Nile tilapia. Naga ICLARM Quarterly, 26 (2): 8-10.

El-Sayed, A.F.M., (2006). Tilapia Culture. CAB International, Wallingford, UK., pp: 304.

FAO, Food and Agriculture Organization of the United Nations, (2010). The State of World Fisheries and Aquaculture 2010. Rome. 197pp.

GAFRD (2011). General Authority for Fish Resources Development yearbook, " Fish Statistics Book in 2011", Ministry of Agriculture and Land Reclamation, Cairo, Egypt, 106 pp.

Hounzangbe-Adote, S.; I. Fouraste ; K. Moutairou and H, Hoste (2005). In vitro effects of four tropical plants on the activity and development of the parasitic nematode, Trichostrongylus colubriformis. J. Helminthol., 79(1): 29-33.

Jackwheeler, M.N. (2003). Health mate Papaya. $\underline{\text { http://www.Papaya aspx.htm. }}$. Jauncey, K. (1998). Tilapia feeds and feeding. Pisces Press Ltd., Stirling,
Scotland. 240p. 
Jegede, T. (2009). Effects of Aloe vera (Liliaceae) on the gonad development in Nile tilapia Oreochromis niloticus (Linnaeus 1758). Better science, better fish, better life, Proceedings of the Ninth International Symposium on Tilapia in Aquaculture. Shanghai Ocean University, Shanghai, China: 22-24.

Jegede, T. (2010). Control of reproduction in Oreochromis niloticus (linnaeus 1758) using hibiscus rosa-sinensis (linn.) leaf meal as reproduction inhibitor. Journal of Agricultural Science, 2 (4):149- 154.

Jegede, T. and O. Fagbenro (2008). Histology of gonads in Oreochromis niloticus (trewavas) fed pawpaw (Carica papaya) seed meal diets. $8^{\text {th }}$ International Symposium on Tilapia in Aquaculture, 1135 - 1141.

Kermanshai, R.; B.E. McCarry; J. Rosenfeld; P.S. Summers; E.A. Weretilnyk and G.J. Sorger (2001). Benzyl isothiocyanate is the chief or sole anthelmintic in papaya seed extracts. Phytochemistry, 57 (3):427-435.

Leite, A.A.; R.M. Nardi; J.R. Nicoli; E. Chartone-Souza and A.M. Nascimento (2005). Carica papaya seed macerate as inhibitor of conjugative R plasmid transfer from Salmonella typhimurium to Escherichia coli, in vitro and in the digestive tract of genotobiotic mice, J. Gen. Appl. Microbiol., 51(1): 21-26.

Levic, J.; M. Sinisa; O. Djuragic and S. Slavica (2008). Herbs and organic acids as an alternative for antibiotic- growth-promoters. Archiva Zootechnica 11: 5-11.

Lohiya, N.K.; B. Manivannan and P.K. Mishra (2002). Chloroform extract of Carica papaya seeds induces long-term reversible azoospermia in Langur monkey. Asian J. Androl., 4: 17-26.

Madhuri, S.; A.K. Mandloi; P. Govind and Y.P. Sahni (2012). Antimicrobial activity of some medicinal plants against fish pathogens. Tnt. Res. J. Pharm., 3(4):28-30.

Niragh, J.O. and M.S. Simmone (1984). Toxic Contaminants in the Great Lakes. John Wiley and Sons, New York, USA.,pp: 335-340.

NRC (National Research Council) (1993). Nutrient requirements of fish. Committee on Animal Nutrition Board on Agriculture. National Academy Press, Washington DC., USA. 114pp.

Osato, J.A.; L.A. Santiago; G.M. Remo; M.S. Cuadra and A. Mori (1993). Antimicrobial and antioxidant activities of unripe papaya, Life Sci., 53(17): 1383-1389.

Pandey, G.; S. Madhuri and A.K. Mandloi (2012). Medicinal plants useful in fish diseases. P1 Arch, 12 (1):1-4.

Passera, C. and P. Spettoli (1981). Chemical composition of papaya seeds. Qual Plant Foods Hum Nutr., (31): 77-83.

Popma, T.J. and L.L. Lovshin (1995). Worldwide prospects for commercial production of tilapia. International Center for Aquaculture and Aquatic Environments Department of Fisheries and Allied Aquacultures Auburn University, Alabama 36849.

SAS. (2006). SAS procedure user's guide. SAS Institute Inc., Cary, NC, USA. Weatherly, A.H. and H.S. Gills (1987). Tissues and Growth. In: The Biology of Fish Growth, Weatherly, A.H., H.S. Gills and J.M. Casselman (Eds.). St. Elmundsbury Press, Great Britain, pp: 147-173. 


$$
\begin{aligned}
& \text { مسحوق بذور الباباظ فى علائق البلطى النيلى الإلى الإنى }
\end{aligned}
$$

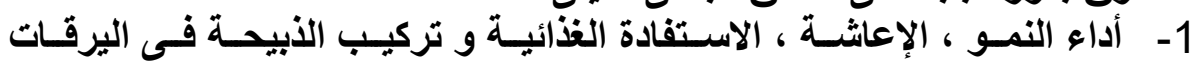

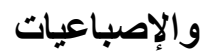

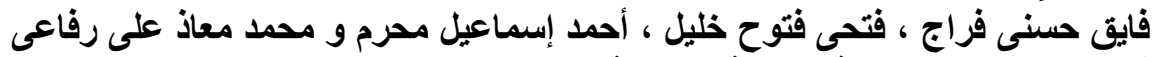

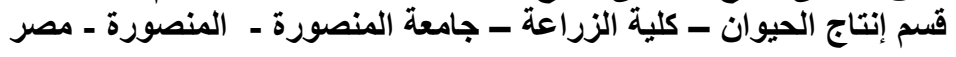

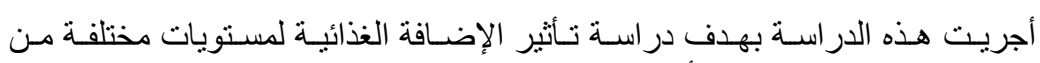

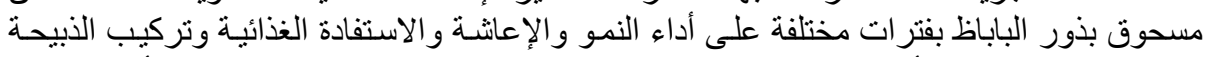

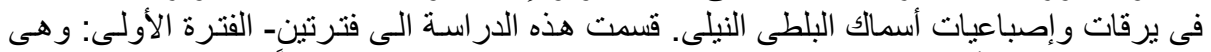

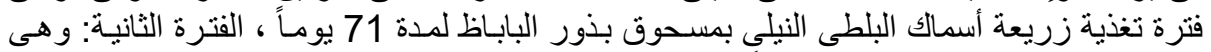

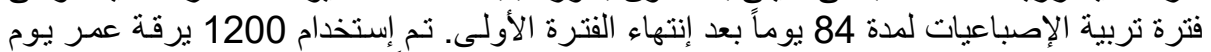

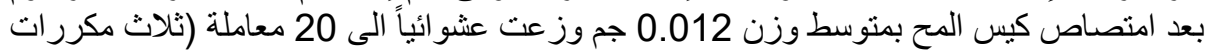

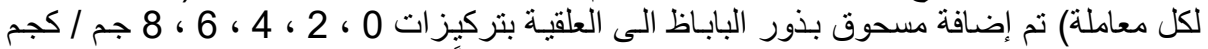

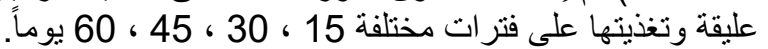

$$
\begin{aligned}
& \text { أشارات نتائج فترة المعاملة الى انها: }
\end{aligned}
$$

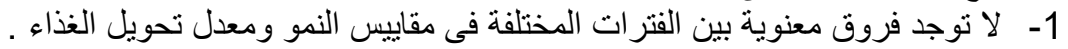

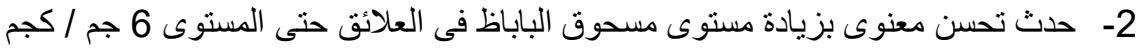

$$
\begin{aligned}
& \text { عليقة ثم انخفضت بالزيادة عن هذا مناد المستوى. }
\end{aligned}
$$

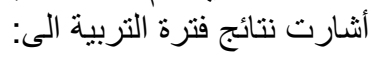

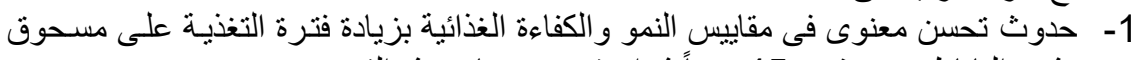

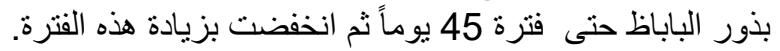

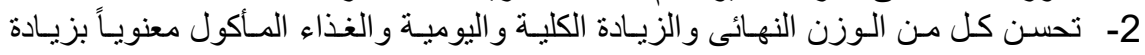

$$
\begin{aligned}
& \text { مستوى مسحوق بذور الباباظ فى العليقة. }
\end{aligned}
$$

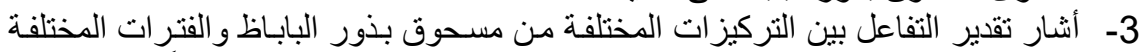

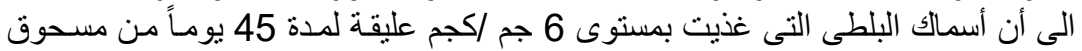

$$
\begin{aligned}
& \text { بذور البابـاظ أعطت أعلى القيم للوزن النهائى و الزيـادة الكليـة واليوميـة مقارنـة بيقيـة }
\end{aligned}
$$

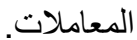

